\title{
ON THE RANGE OF AN INVARIANT MEAN
}

\author{
BY \\ E. E. GRANIRER
}

Introduction. Let $S$ be a discrete semigroup, $m(S)$ the space of bounded real functions on $S$ with the usual sup. norm, and $m(S)^{*}$ the conjugate Banach space of $m(S)$. An element $\phi \in m(S)^{*}$ is a mean if $\phi(f) \geqq 0$ whenever $f \geqq 0$ and $\phi(1)=1$, where 1 denotes also the constant one function on $S$.

$S$ is said to be left [right] amenable if there is a mean $\phi \in m(S)^{*}$ which is in addition left [right] invariant, i.e., satisfies $\phi\left(f_{a}\right)=\phi(f)\left[\phi\left(f^{a}\right)=\phi(f)\right]$ for each $f \in m(S)$ and $a \in S$ (where $f_{a}(s)=f(a s)$ and $f^{a}(s)=f(s a)$ for $f$ in $m(S)$ and $a, s \in S$ ). $S$ is amenable if there is a mean $\phi \in m(S)^{*}$ which is left and right invariant.

If $A \subset S$, then $1_{A}$ will be the function which is one on $A$ and zero otherwise. We shall write 1 instead of $1_{S}$ and $\phi(A)$ instead of $\phi\left(1_{A}\right)$ (if $\left.\phi \in m(S)^{*}\right)$, sometimes.

The range of an element $\phi \in m(S)$ is the set of numbers $\{\phi(A)$, where $A$ ranges over all subsets of $S\}$. It is clear that the range of a mean is a subset of $[0,1]$ $=\{x ; 0 \leqq x \leqq 1\}$.

If $S$ is a left amenable semigroup, define the following relation between elements of $S: a \sim b$ iff $a s=b s$ for some $s$ in $S$. The relation $\sim$ is an equivalence relation which is two-sidedly stable (or a congruence), i.e., if $a \sim b$, then $a s \sim b s$ and $s a \sim s b$ for any $s$ in $S$ (since $S$ is left amenable) (see [3, p. 371] and Ljapin [1, p. 39]).

If $s \in S$, let $s^{\prime}$ stand for the equivalence class to which $s$ belongs and let $S^{\prime}=\left\{s^{\prime} ; s \in S\right\}$. A multiplication between the elements of $S^{\prime}$ can be defined by $s^{\prime} t^{\prime}=(s t)^{\prime}$. This multiplication is well defined and associative, rendering thus $S^{\prime}$ a semigroup (since $\sim$ is a congruence. See Ljapin [1, pp. 265-266]). Furthermore, $S^{\prime}$ has right cancellation (and coincides with $S$ if $S$ has right cancellation).

It has been shown by this author in [4] (Corollary to Lemma 1) that, if $S$ has right cancellation, is left amenable and contains an element of infinite order, then the range of any invariant mean on $m(S)$ contains the set of rationals in $[0,1]$. Moreover, if $0 \leqq r \leqq 1$ is a rational number, then there is a set $A \subset S$ such that $\phi(A)=r$ for any left invariant mean $\phi$ on $m(S)$.

T. Mitchell has even suggested orally a proof to show that, for the above considered semigroup $S$, the range of each left invariant mean $\phi$ on $m(S)$ is the whole $[0,1]$ interval.

We would like to prove in this paper the following:

Conjecture. Let $S$ be a left amenable semigroup. Then the range of each left invariant mean on $m(S)$ is the whole $[0,1]$ interval if and only if $S^{\prime}$ is infinite.

Received by the editors April 6, 1966. 
Unfortunately, we do not know how to prove this result. But we know how to prove something close to it.

Call a group $G$ an $A B$ group if: (a) $G$ is amenable, (b) each element of $G$ has finite order, (c) each infinite subgroup of $G$ is not locally finite (a group is locally finite if each finitely generated subgroup is finite), (d) $G$ is an infinite group.

We doubt whether $A B$ groups exist at all, since if $G$ were an $A B$ group, then each of its infinite subgroups would contain a subgroup which would provide a counterexample to Burnside's conjecture and would be in addition, amenable.

The main result of this paper is the

THeOREM 3. Let $S$ be a left amenable semigroup for which $S^{\prime}$ is not an AB group. Then the range of each left invariant mean on $m(S)$ is the whole $[0,1]$ interval if and only if $S^{\prime}$ is infinite.

It comes out that if $S^{\prime}$ is not an $A B$ group, then $m(S)$ admits a left invariant mean whose range is not the whole $[0,1]$ interval, if and only if $S^{\prime}$ is a finite group. In this case, there is even a left invariant mean on $m(S)$ (which may annihilate single point sets) whose range is the set $\{k / n ; k=0,1, \ldots, n\}$, where $n$ is the order of $S^{\prime}$.

We prove in fact, more than stated in the above theorem, namely that if $S^{\prime}$ is an infinite non- $A B$ group, then there is a class of subsets of $S\{A(x), 0 \leqq x \leqq 1\}$ which satisfies $A(0)=\varnothing, A(1)=S, A(x) \subset A\left(x^{\prime}\right)$ if $0 \leqq x \leqq x^{\prime} \leqq 1$ and $\phi(A(x))=x$ for each left invariant mean $\phi \in m(S)^{*}$ and each $0 \leqq x \leqq 1$. In particular, the range of a left invariant mean is attained on the class of sets $A \subset S$, for which $\phi(A)$ is constant when $\phi$ ranges over the set of left invariant means.

In conclusion, it is a pleasure to thank $T$. Mitchell for the friendly and fruitful conversations we had with him. The idea of the proof of Theorem 1 and of the lemma preceding it, was basically suggested by him.

Some more notations. Let $S$ be a semigroup. If $a \in S$, we denote by $l_{a}: m(S) \rightarrow$ $m(S)$, defined by $l_{a} f=f_{a}$ for $f$ in $m(S)$. If $\phi \in m(S)$, then $L_{a}: m(S)^{*} \rightarrow m(S)^{*}$ is defined by $\left(L_{a} \phi\right) f=\phi\left(f_{a}\right)$ for each $f$ in $m(S)$. If $A \subset S$ we say that $\phi \in m(S)^{*}$ is $A$-left invariant if $L_{a} \phi=\phi$ for each $a$ in $A$. If $A$ consists of the single element $c \in S$, then we say that $\phi$ is $c$-left invariant. If $A, B$ are subsets of $S$, then $B$ is $A$-left almost convergent if $\phi_{1}(B)=\phi_{2}(B)$ for any two $A$-left invariant means $\phi_{1}$, $\phi_{2} \in m(S)^{*} . B \subset S$ is left almost convergent if it is $S$-left almost convergent. For characterizations of almost convergent subsets of the additive semigroup of positive integers, see G. Lorentz [6] (see also Day [2, pp. 538-540]).

If $S$ is a right cancellation semigroup, then $c \in S$ has order $n \geqq 1$, if $n+1$ is the first integer which satisfies $c^{n+1}=c$. $c \in S$ has infinite order if no such $n \geqq 1$ exists.

\section{The main theorem.}

LEMMA 1a. Let $S$ be a right cancellation semigroup, $c \in S$ an element of infinite 
order, and $A \subset S$ be such that $c A \subset A$. Let $Q_{n}=\left\{p / 2^{n} ; p=0,1, \ldots, 2^{n}\right\}$ and $Q=\bigcup_{n=1}^{\infty} Q_{n}$. Then there exists a collection of sets $A(r), r \in Q$, such that

(1) $A(0)=\varnothing, A(1)=A$.

(2) If $r, r^{\prime} \in Q$ with $r<r^{\prime}$, then $A(r) \subset A\left(r^{\prime}\right)$.

(3) $\phi(A(r))=r \phi(A)$ for any c-left invariant mean $\phi$.

Proof. By Lemma 1 of [4], there are disjoint sets $V_{1}, V_{2} \subset A$ such that $A$ $=V_{1} \cup V_{2}, c V_{1} \subset V_{2}$ and $c V_{2} \subset V_{1}$ (hence $c^{2} V_{1} \subset V_{1}$ ). Let $A(0)=\varnothing, A\left(\frac{1}{2}\right)=V_{1}$, and $A(1)=A=V_{1} \cup V_{2}$. Then

$$
c^{2}\left[A\left(\frac{1}{2}\right)-A(0)\right] \subset\left[A\left(\frac{1}{2}\right)-A(0)\right], \quad c^{2}\left[A(1)-A\left(\frac{1}{2}\right)\right] \subset\left[A(1)-A\left(\frac{1}{2}\right)\right],
$$

and if $\phi$ is any $c$-left invariant mean, then $\phi\left(V_{1}\right) \leqq \phi\left(c V_{1}\right) \leqq \phi\left(V_{2}\right) \leqq \phi\left(c V_{2}\right) \leqq \phi\left(V_{1}\right)$, i.e., $\phi\left(V_{1}\right)=\phi\left(V_{2}\right)$. Hence, $\phi\left(A\left(\frac{1}{2}\right)\right)=\frac{1}{2} \phi(A)$ and so $\phi(A(r))=r \phi(A)$ for any $r \in Q_{1}$. For fixed $n$, arrange the set of rationals $Q_{n}$ in increasing order as $0=r_{0}<r_{1}<\cdots$ $<r_{2^{n}}=1$ and assume that we have found sets $A(r) \subset A, r \in Q_{n}$ such that $A(r) \subset A\left(r^{\prime}\right)$ if $r, r^{\prime} \in Q_{n}$ with $r<r^{\prime}, A(0)=\varnothing, A(1)=A$ and such that $\phi(A(r))=r \phi(A)$ for any $c$-left invariant mean $\phi$ and any $r \in Q_{n}$ and such that for some $k>0$,

$$
c^{k}\left[A\left(r_{i}\right)-A\left(r_{i-1}\right)\right] \subset\left[A\left(r_{i}\right)-A\left(r_{i-1}\right)\right]
$$

for $1 \leqq i \leqq 2^{n}$.

There are then, by Lemma 1 of [4], disjoint sets $V_{1}^{(i)}, V_{2}^{(i)}$ with

$$
V_{1}^{(i)} \cup V_{2}^{(i)}=A\left(r_{i}\right)-A\left(r_{i-1}\right),
$$

$c^{k} V_{1}^{(i)} \subset V_{2}^{(i)}$, and $c^{k} V_{2}^{(i)} \subset V_{1}^{(i)}$. If $\phi$ is any $c$-left invariant mean then as before $\phi\left(V_{1}^{(i)}\right)=\phi\left(V_{2}^{(i)}\right)$ and so

$$
2 \phi\left(V_{1}^{(i)}\right)=\phi\left[A\left(r_{i}\right)-A\left(r_{i-1}\right)\right]=\left(r_{i}-r_{i-1}\right) \phi(A)=\phi(A) / 2^{n} .
$$

Hence $\phi\left(V_{1}^{(i)}\right)=\phi(A) / 2^{n+1}$. Define now: $A\left(r_{i-1}+1 / 2^{n+1}\right)=A\left(r_{i-1}\right) \cup V_{1}^{(i)}$ for $1 \leqq i \leqq 2^{n}$. Since $V_{1}^{(i)} \cap A\left(r_{i-1}\right)=0$, we have that

$$
\phi(A)\left(r_{i-1}+1 / 2^{n+1}\right)=\left(r_{i-1}+1 / 2^{n+1}\right) \phi(A)
$$

for any $c$-left invariant mean. For $r \in Q_{n+1}$ and $r \notin Q_{n}$ we have thus defined sets $A(r)$. If $r \in Q_{n}$, let $A(r)$ be given by the induction hypothesis. (Hence the sets $A(r)$ constructed at stage $n+1$ coincide for $r \in Q_{n}$ with the sets built at stage $n$.) We have, from above, that $A\left(r_{i}\right)-A\left(r_{i-1}+1 / 2^{n+1}\right)=V_{2}^{(i)}$ and $A\left(r_{i-1}+1 / 2^{n+1}\right)-A\left(r_{i-1}\right)=V_{1}^{(i)}$. Hence, if the elements of $Q_{n+1}$ are arranged in increasing order as $0=s_{0}<s_{1}<\cdots$ $<s_{2^{n+1}}$, then $c^{2 k}\left[A\left(s_{i}\right)-A\left(s_{i-1}\right)\right] \subset A\left(s_{i}\right)-A\left(s_{i-1}\right), A\left(s_{i-1}\right) \subset A\left(s_{i}\right)$ for $1 \leqq i \leqq 2^{n+1}$ and $\phi\left(A\left(s_{i}\right)\right)=s_{i} \phi(A)$ for any $c$-left invariant mean. Consider now the sets $A(r)$, $r \in Q=\bigcup^{\infty} Q_{n}$. If $r, r^{\prime} \in Q$ with $r<r^{\prime}$, then $r, r^{\prime} \in Q_{n}$ for some $n$ (since $Q_{n} \subset Q_{n+1}$ ); hence $A(r) \subset A\left(r^{\prime}\right)$. Conditions (1), (3) are satisfied from above. 
THEOREM 1. Let $S$ be a right cancellation semigroup, $c \in S$ an element of infinite order, and $A \subset S$ be such that $c A \subset A$. Then there exists a collection of sets $\{A(x) ; 0 \leqq x \leqq 1\}$ such that:

(1) $A(0)=\varnothing, A(1)=A$.

(2) If $0 \leqq x<x^{\prime} \leqq 1$, then $A(x) \subset A\left(x^{\prime}\right)$.

(3) $\phi(A(x))=x \phi(A)$ for any c-left invariant mean $\phi$ on $m(S)$ and any $x \in[0,1]$.

Proof. For $x \in Q=\bigcup_{n=1}^{\infty} Q_{n}$, let $A(x)$ be the sets constructed in the previous lemma. If $0<x<1$ is such that $x \notin Q$, define $A(x)=\bigcap_{\{r \in Q: r>x\}} A(r)$. If now $0 \leqq x<x^{\prime} \leqq 1$, then:

(a) if $x \in Q$, then $A(x) \subset A(r)$ for any $r>x^{\prime}$; hence $A(x) \subset A\left(x^{\prime}\right)$,

(b) if $x^{\prime} \in Q$, then $A(x) \subset A\left(x^{\prime}\right)$ is clear, and

(c) if $x \notin Q$ and $x^{\prime} \notin Q$, then for some $r \in Q$ we have $x<r<x^{\prime}$ which implies $A(x) \subset A(r) \subset A\left(x^{\prime}\right)$. Hence, (1) and (2) hold.

If now $0<x<1$ and $x \notin Q$ and $\phi$ is a $c$-left invariant mean, then

$$
r \phi(A)=\phi(A(r)) \leqq \phi(A(x)) \leqq \phi\left(A\left(r^{\prime}\right)\right)=r^{\prime} \phi(A)
$$

for any $r, r^{\prime} \in Q$ with $r<x<r^{\prime}$. This implies that $x(\phi(A)) \leqq \phi(A(x)) \leqq x \phi(A)$ since $Q$ is dense in $[0,1]$, which finishes this proof.

COROLlaRy. Let $S$ be a right cancellation semigroup, $c \in S$ an element of infinite order. There exists a class of sets $\{A(x) ; 0 \leqq x \leqq 1\}$ such that:

(1) $A(0)=\varnothing$ and $A(1)=S$.

(2) $A(x) \subset A\left(x^{\prime}\right)$ if $0 \leqq x<x^{\prime} \leqq 1$.

(3) $\phi(A(x))=x$ for any $0 \leqq x \leqq 1$ and any c-left invariant mean $\phi \in m(S)^{*}$.

The idea of using the background of [4] (see [4, Lemma 1 and Corollary to Lemma 1]) as above and especially the use of the monotonicity of an invariant mean as in the proof of Theorem 1 is due to T. Mitchell (oral communication).

Remarks. Let $X$ be a set, $\mathscr{A}$ an algebra of subsets of $X$ and $v$ a finitely additive, finite measure on $\mathscr{A}$. Then $\nu$ has the intermediate property on $A \in \mathscr{A}$ if for any $0 \leqq b \leqq \nu(A)$ there exists a $B \in \mathscr{A}$ such that $B \subset A$ and $\nu(B)=b$. One has for the above $S$ that a $c$-left invariant mean has the intermediate property on any $A \subset S$ for which $c A \subset A$. Moreover, if $A$ is $c$-left almost convergent, there is even a set $B \subset A$ which is $c$-left almost convergent for which $\phi(B)=b$.

As a consequence of the above corollary, one has that, if $S$ is a right cancellation left amenable semigroup, (and in particular an amenable group) which contains an element of infinite order, then the range of any left invariant mean $\phi$ is the whole $[0,1]$ interval and, moreover, $A \subset S$ need only range in the class of left almost convergent subsets of $S$ in order that $\phi(A)$ should fill the entire $[0,1]$ interval.

We ask now what is the range of a left invariant mean when $S$ is a group which is the full direct product of countably many copies of the multiplicative group $\{-1,1\}$. This group is infinite and each of its elements has order two. The above 
theorem does not answer this question. Another question is what is the range of a left invariant mean on the group of all the permutations of the integers which leave all but a finite number of integers fixed. Both these groups are locally finite infinite groups. We shall show that if $G$ is any amenable group which contains a locally finite infinite group, then the range of any left invariant mean $\phi$ on $m(G)$ is the whole $[0,1]$ interval. We show even more than that in Theorem 2.

Notation. $G$ will denote a discrete group with identity $e$. If $H \subset G$ is a subgroup of $G$ then $G / H$ will always stand for the set of right cosets of $G$ with respect to $H$, i.e., for $\{H g ; g \in G\}$. We say then that $A=\left\{g_{\alpha} ; \alpha \in I\right\} \subset G$ is a set of representatives of $G / H$ if $G=H A$ and $H g_{\alpha} \cap H g_{\beta} \neq \varnothing$ implies $g_{\alpha}=g_{\beta}$. We do not speak at all in what follows about left cosets. If $A_{1}, \ldots, A_{n}$ are subsets of $G$, then

$$
A_{1} \cdots A_{n}=\left\{a_{1} a_{2} \cdots a_{n} ; a_{i} \in A_{i}, i=1,2, \ldots, n\right\} .
$$

RemarK 1. Let $H \subset H_{1}$ be subgroups of the group $H_{2}$ and let $A_{1}, A_{2}$ be sets of representatives of $H_{1} / H, H_{2} / H_{1}$, respectively. Then $A_{1} A_{2}$ is a set of representatives of $H_{2} / H$. Since $H_{1}=H A_{1}$ and $H_{2}=H_{1} A_{2}=H\left(A_{1} A_{2}\right)$ and if $H a_{1} a_{2} \cap H a_{1}^{\prime} a_{2}^{\prime} \neq \varnothing$ with $a_{i}, a_{i}^{\prime} \in A_{i}, i=1,2$, then $H_{1} a_{2} \cap H_{1} a_{2}^{\prime} \neq \varnothing$; hence $a_{2}=a_{2}^{\prime}$ which implies that $H a_{1} \cap H a_{1}^{\prime} \neq \varnothing$. Therefore, $a_{1}=a_{1}^{\prime}$. In particular, $a_{1} a_{2}=a_{1}^{\prime} a_{2}^{\prime}$ with $a_{i}, a_{i}^{\prime} \in A_{i}$, $i=1,2$, is possible if and only if $a_{1}=a_{1}^{\prime}$ and $a_{2}=a_{2}^{\prime}$.

LEMMA 1. Let $S_{n}$ be subgroups of $G$ such that $S_{n} \subset S_{n+1}$ for $n=1,2, \ldots$ Let $R_{n}$ be a set of representatives of $S_{n} / S_{n-1}$ with $e \in R_{n}$ for each $n$ and $S=\bigcup_{n=1}^{\infty} S_{n}$. Then $U_{j}=\bigcup_{n=j+1}^{\infty}\left\{R_{j+1} \cdots R_{n}\right\}$ is a set of representatives of $S / S_{j}$ for $j=1,2, \ldots$

Proof. $\left\{R_{j+1} \cdots R_{n}\right\}$ is a set of representatives of $S_{n} / S_{j}$ for $n \geqq j+1$. Since, by definition, $R_{j+1}$ is a set of representatives of $S_{j+1} / S_{j}$ and if $\left\{R_{j+1} \cdots R_{n-1}\right\}$ is a set of representatives of $S_{n-1} / S_{j}$ then, by Remark $1,\left\{R_{j+1} \cdots R_{n-1} R_{n}\right\}$ is a set of representatives of $S_{n} / S_{j}$. Furthermore,

$$
S=\bigcup_{n=j+1}^{\infty} S_{n}=\bigcup_{n=j+1}^{\infty}\left\{S_{j} R_{j+1} \cdots R_{n}\right\}=S_{j}\left[\bigcup_{n=j+1}\left\{R_{j+1} \cdots R_{n}\right\}\right]=S_{j} U_{j} .
$$

Assume now that $S_{j} r \cap S_{j} r^{\prime} \neq \varnothing$ for $r \in R_{j+1} \cdots R_{n}, r^{\prime} \in R_{j+1} \cdots R_{m}$. (We can assume that $n \geqq m$.) Since $e \in R_{i}$ for each $i, R_{j+1} \cdots R_{k} \subset R_{j+1} \cdots R_{k} R_{k+1}$. Hence, $r, r^{\prime} \in R_{j+1} \cdots R_{n}$ which is a set of representatives $S_{n} / S_{j}$. Therefore, $r=r^{\prime}$ which finishes this proof.

REMARK 2. In the notation of Lemma 1 , let $R$ be a set of representatives of $G / S$ and define

$$
V_{j}=\bigcup_{n=j+1}^{\infty}\left\{R_{j+1} \cdots R_{n}\right\} R=U_{j} R .
$$

Then $V_{j}$ is a set of representatives of $G / S_{j}$ (apply Remark 1). Furthermore, from the definition of $V_{j}$, it follows immediately that $R_{j} V_{j}=V_{j-1}$ for $j \geqq 2$ (which is a set of representatives of $\left.G / S_{j-1}\right)$. 
REMARK 3. If $H$ is a subgroup of $G$ and $V$ a set of representatives of $G / H$, then clearly $H V=G$ and $h V \cap h^{\prime} V \neq \varnothing$, with $h, h^{\prime} \in H$, implies that $h=h^{\prime}$. Since if $h v=h^{\prime} v^{\prime}$ with $v, v^{\prime} \in V$, then $H v \cap H v^{\prime} \neq \varnothing$, i.e., $v=v^{\prime}$ and so $h=h^{\prime}$.

LEMMA 2. The above chosen subsets $V_{1}, V_{2}, \ldots$ of $G$ satisfy:

(1) $V_{n} \supset V_{n+1}$ for each $n \geqq 1$.

(2) $S_{j} V_{j}=G$ and $s V_{j} \cap s^{\prime} V_{j} \neq \varnothing$ with $s, s^{\prime} \in S_{j}$ implies $s=s^{\prime}$.

(3) $R_{j} V_{j}=V_{j-1}$ for $j \geqq 2$.

Proof. (1) and (2) are clear from Remarks 2 and 3 while $R_{j} V_{j}=V_{j-1}$ by definition.

LEMMA 3. Let $S_{n}$ be finite subgroups of $G$ of order $p_{n}<\infty$ such that $S_{n} \subset S_{n+1}$ (proper inclusion) for $n=1,2, \ldots$ Let $S=\bigcup_{1}^{\infty} S_{n}, Q_{n}=\left\{k / p_{n} ; k=0,1, \ldots, p_{n}\right\}$, and $Q=\bigcup_{1}^{\infty} Q_{n}$. Then there exists a family of subsets of $S,\{A(r) ; r \in Q\}$ such that:

(1) $A(0)=\varnothing, A(1)=G$.

(2) If $r_{1}<r_{2}$ with $r_{1}, r_{2} \in Q$, then $A\left(r_{1}\right) \subset A\left(r_{2}\right)$.

(3) $\phi(A(r))=r$ for each $r \in Q$ and each $S$-left invariant mean $\phi \in m(G)^{*}$.

Proof. Choose for each $n$ a fixed set $R_{n}$ of representatives of $S_{n} / S_{n-1}$ with $e \in R_{n}$. Since $S_{n}$ is a proper subgroup of $S_{n+1}$, we have that $p_{n} q_{n}=p_{n+1}$ for some $q_{n} \geqq 2$ and $R_{n}$ contains $q_{n-1}$ elements. Furthermore, $Q_{n} \subset Q_{n+1}$ for each $n$ (since $k / p_{n}=k q_{n}\left(p_{n+1}\right)$. Consider the sets $V_{n}$ constructed in Lemma 3 with respect to the $R_{n}$ 's. We construct the sets $A(r)$ for $r \in Q_{1}$ as follows: Assume that

$$
S_{1}=\left\{s_{1}^{(1)}, s_{2}^{(1)}, \ldots, s_{p_{1}}^{(1)}\right\}
$$

is an enumeration of the elements of $S_{1}$ with $s_{1}^{(1)}=e$. Let then: $A(0)=\varnothing$, $A\left(1 / p_{1}\right)=V_{1}, \quad A\left(2 / p_{1}\right)=V_{1} \cup s_{2}^{(1)} V_{1}, \ldots, \quad A\left(k / p_{1}\right)=V_{1} \cup s_{2}^{(1)} V_{1} \cup \cdots \cup s_{k}^{(1)} V_{1}$ for $k=1,2, \ldots, p_{1}$.

Since $S_{1} V_{1}=G$ and $s V_{1} \cap s^{\prime} V_{1} \neq \varnothing$ if $s \neq s^{\prime}$ with $s, s^{\prime} \in S_{1}$ we have that $\phi\left(V_{1}\right)$ $=1 / p_{1}$ and $\phi\left(A\left(k / p_{1}\right)\right)=k / p_{1}$ if $k=0,1, \ldots, p_{1}$ for each $S$-left invariant mean $\phi$. Also, $A\left(r_{1}\right) \subset A\left(r_{2}\right)$ if $r_{1}<r_{2}$ and $r_{1}, r_{2} \in Q_{1}$.

Assume now that sets $A(r)$ have been built for $r \in Q_{n}$ such that $A\left(1 / p_{n}\right)=V_{n}$, $A(0)=\varnothing$, and such that for some enumeration of the elements of $S_{n}$, say $S_{n}$ $=\left\{s_{1}^{(n)}, \ldots, s_{p_{n}}^{(n)}\right\}$ with $s_{1}^{(n)}=e, A\left(k / p_{n}\right)=\bigcup_{i=1}^{k} s_{i}^{(n)} V_{n}$ if $1 \leqq k \leqq p_{n}$. We define then sets $A(r), r \in Q_{n+1}$, as follows: If $k=i q_{n}$ for $i=0,1, \ldots, p_{n}$, then $k / p_{n+1}=i / p_{n}$. (We define in this case $A\left(k / p_{n+1}\right)=A\left(i / p_{n}\right)$ where $A\left(i / p_{n}\right)$ is given by the induction hypothesis.) We define $A\left(1 / p_{n+1}\right)=V_{n+1}$ and if $R_{n+1}=\left\{t_{1}, \ldots, t_{q_{n}}\right\}$ with $t_{1}=e$, define

$$
A\left(\frac{k}{p_{n+1}}\right)=\bigcup_{i=1}^{k} t_{i} V_{n+1} \quad \text { for } 1 \leqq k \leqq q_{n} .
$$

(Since $R_{n+1} V_{n+1}=V_{n}$, we have that $A\left(q_{n} / p_{n+1}\right)=A\left(1 / p_{n}\right)$ in agreement with the previous definition of $A\left(1 / p_{n}\right)$.) We define:

$$
\begin{aligned}
A\left(\frac{k}{p_{n}}+\frac{j}{p_{n+1}}\right) & =A\left(\frac{k}{p_{n}}\right) \cup s_{k+1}^{(n)} A\left(\frac{j}{p_{n+1}}\right) \\
& \text { for } 1 \leqq j \leqq q_{n}-1 \text { and } k=1,2, \ldots, p_{n}-1 .
\end{aligned}
$$


We have

$$
\begin{aligned}
A\left(\frac{k}{p_{n}}+\frac{j}{p_{n+1}}\right) & =\left[\bigcup_{i=1}^{k} s_{i}^{(n)} V_{n}\right] \cup\left[s_{k+1}^{(n)}\left[t_{1} V_{n+1} \cup \cdots \cup t_{j} V_{n+1}\right]\right] \\
& =\left[\bigcup_{i=1}^{k} \bigcup_{m=1}^{q_{n}} s_{i}^{(n)} t_{m} V_{n+1}\right] \cup\left[s_{k+1}^{(n)}\left[t_{1} V_{n+1} \cup \cdots \cup t_{j} V_{n+1}\right]\right]
\end{aligned}
$$

if $1 \leqq j \leqq q_{n}-1$ and $1 \leqq k \leqq p_{n}-1$.

Enumerate now the elements of $S_{n+1}$ by:

$$
s_{1}^{(n+1)}=t_{1}=e, s_{2}^{(n+1)}=t_{2}, \ldots, s_{q_{n}}^{(n+1)}=t_{q_{n}}, s_{i q_{n}}^{(n+1)}=s_{i}^{(n)} t_{q_{n}}
$$

for $i=1,2, \ldots, p_{n}$, and

$$
s_{i q_{n}+j}^{(n+1)}=s_{i+1}^{(n)} t_{j} \quad \text { if } 1 \leqq j \leqq q_{n}-1 \quad \text { and } \quad 0 \leqq i \leqq p_{n}-1 .
$$

It follows from $(*)$, upon a moment's reflection, that

$$
\begin{aligned}
A\left(\frac{k q_{n}+j}{p_{n+1}}\right) & =A\left(\frac{k}{p_{n}}+\frac{j}{p_{n+1}}\right)=\left[\bigcup_{i=1}^{k q_{n}} s_{i}^{(n+1)} V_{n+1}\right] \cup s_{k q_{n}+1}^{(n+1)} V_{n+1} \cup \cdots \cup s_{k q_{n}+j}^{(n+1)} V_{n+1} \\
& =\bigcup_{i=1}^{k q_{n}+j} s_{i}^{(n+1)} V_{n+1} \quad \text { if } 0 \leqq k \leqq p_{n}-1 \text { and } \quad 1 \leqq j \leqq q_{n}-1
\end{aligned}
$$

Furthermore,

$$
\begin{aligned}
A\left(\frac{k q_{n}}{p_{n+1}}\right) & =A\left(\frac{k}{p_{n}}\right)=\bigcup_{i=1}^{k} s_{i}^{(n)} V_{n}=\bigcup_{i=1}^{k} \bigcup_{j=1}^{q_{n}} s_{i}^{(n)} t_{j} V_{n+1} \\
& =\bigcup_{m=1}^{k q_{n}} s_{m}^{(n+1)} V_{n+1} \quad \text { for } k=1,2, \ldots, p_{n} .
\end{aligned}
$$

We have thus constructed sets $A(r), r \in Q_{n+1}$, which coincide with the sets $A(r)$ given by the induction hypothesis for $r \in Q_{n}$, such that $A\left(1 / p_{n+1}\right)=V_{n+1}$ and for some enumeration of $S_{n+1}=\left\{s_{1}^{(n+1)}, \ldots, s_{p_{n+1}}^{(n+1)}\right\}$, with $s_{1}^{(n+1)}=e$, we have that

$$
A\left(\frac{k}{p_{n+1}}\right)=\bigcup_{i=1}^{k} s_{i}^{(n+1)} V_{n+1} \quad \text { for } 1 \leqq k \leqq p_{n+1} .
$$

We can hence assume that there exists a class of subsets of $G,\{A(r) ; r \in Q\}$ such that $A(0)=\varnothing, A(1)=G$ and such that for each $n$ there is an enumeration of $S_{n}$, $S_{n}=\left\{s_{1}^{(n)}, \ldots, s_{p_{n}}^{(n)}\right\}$ with $e=s_{1}^{(n)}$ such that $A\left(k / p_{n}\right)=\bigcup_{i=1}^{k} s_{i}^{(n)} V_{n}$ for $1 \leqq k \leqq p_{n}$ and $A(0)=\varnothing$. (The delicate point here is that $A(r)$ does not depend on the particular representation of $r$ as $k / p_{n}$.)

If $r, r^{\prime} \in Q$ with $r<r^{\prime}$, say, then $r, r^{\prime} \in Q_{n}$ for some $n$. Hence, $r=k / p_{n}<k^{\prime} / p_{n}=r^{\prime}$ and so $A(r)=\bigcup_{1}^{k} s_{i}^{(n)} V_{n} \subset \bigcup_{1}^{k^{\prime}} s_{i}^{(n)} V_{n}=A\left(r^{\prime}\right)$. Furthermore, by Lemma 2, $s V_{n} \cap s^{\prime} V_{n}$ $=0$ if $s \neq s^{\prime}$ and $s, s^{\prime} \in S_{n}$. Hence, $\phi\left[A\left(k / p_{n}\right)\right]=k \phi\left(V_{n}\right)$ for any $S$-left invariant mean $\phi$. But

$$
\phi(G)=\phi\left[\bigcup_{1}^{p_{n}} s_{i}^{(n)} V_{n}\right]=p_{n} \phi\left(V_{n}\right) ; \text { i.e., } \phi\left(V_{n}\right)=\frac{1}{p_{n}} .
$$

This implies that $\phi\left(A\left(k / p_{n}\right)\right)=k / p_{n}$ or that $\phi(A(r))=r$ for each $r \in Q$ and any $S$-left invariant mean $\phi$ on $m(G)$. This finishes the proof. 
THEOREM 2. Let $S_{n}$ be finite subgroups of $G$ with $S_{n} \subset S_{n+1}$ (proper inclusion) and let $S=\bigcup_{n=1}^{\infty} S_{n}$. Then there exists a class of subsets of $G,\{A(x) ; 0 \leqq x \leqq 1\}$ such that

(1) $A(0)=\varnothing, A(1)=G$.

(2) If $0 \leqq x<x^{\prime} \leqq 1$, then $A(x) \subset A\left(x^{\prime}\right)$.

(3) $\phi(A(x))=x$ for each $0 \leqq x \leqq 1$ and each S-left invariant mean $\phi$ on $m(G)$.

Proof. Let $p_{n}$ be the order of $S_{n}$ and consider the sets $Q_{n}$ and $Q=\bigcup_{1}^{\infty} Q_{n}$ of Lemma 3. $Q$ is dense in $[0,1]$. For $r \notin Q$, choose the sets $A(r)$ constructed in Lemma 3. If $0<x<1$ is such that $x \in Q$, then let $A(x)=\bigcap_{\{r \in Q ; r>x\}} A(r)$. Let $0 \leqq x<x^{\prime} \leqq 1$. If either $x \in Q$ or $x^{\prime} \in Q$, then clearly $A(x) \subset A\left(x^{\prime}\right)$. If $x$ and $x^{\prime}$ are not in $Q$, then let $r \in Q$ be such that $x<r<x^{\prime}$. Then $A(x) \subset A(r) \subset A\left(x^{\prime}\right)$. Let now $\phi$ be any $S$-left invariant mean on $m(G)$ and $0 \leqq x \leqq 1$ with $x \notin Q$.

If $r, r^{\prime} \in Q$ are such that $r<x<r^{\prime}$, then

$$
r^{\prime}=\phi\left(A\left(r^{\prime}\right)\right) \leqq \phi(A(x)) \leqq \phi(A(r))=r .
$$

Since $Q$ is dense in $[0,1], \phi(A(x))=x$, which finishes the proof.

REMARK. If $G$ contains an element $a$ of order two and $\phi$ is a mean whose range is $\{0,1\}$, then $\frac{1}{2}\left(L_{a} \phi+L_{a^{2}} \phi\right)$ is an $a$-left invariant mean whose range is $\left\{0, \frac{1}{2}, 1\right\}$.

Let $S$ be a semigroup. $S$ is said to be periodic if for any $s \in S$ there is some $n \geqq 1$ such that $s^{n}$ is an idempotent (see Ljapin [1, p. 113]). We shall subsequently need the following:

LEMMA 4. Let $S$ be a right cancellation left amenable semigroup which is periodic. Then $S$ is a $\operatorname{group}\left({ }^{1}\right)$.

Proof. Let $E$ be the set of all idempotents of $S$. For $e \in E$, let $R_{e}=\left\{a \in S ; a^{r}=e\right.$ for some $r \geqq 1\}$. If $e_{1}, e_{2} \in E$ and $a^{r_{1}}=e_{1} a^{r_{2}}=e_{2}$ for some $a \in S$, then $e_{1}=a^{r_{1} r_{2}}=e_{2}$. Hence, $R_{e_{1}} \cap R_{e_{2}}=0$ if $e_{1} \neq e_{2}$ and $R_{e} \cap E=\{e\}$ for each $e \in E$. (For this argument, see Ljapin [1, p. 114].)

$R_{e}$ contains a right ideal for each $e \in E$. In fact, if $a \in S$, then $(e a)^{n}$ is an idempotent for some $n \geqq 1$. Hence $(e a)^{2 n}=e(e a)^{n}$ which implies by the right cancellation that $(e a)^{n}=e$. Therefore, $e S \subset R_{e}$. Since $S$ is left amenable, any two right ideals have nonvoid intersection and, therefore,

$$
R_{e_{1}} \cap R_{e_{2}} \neq \varnothing \quad \text { for } e_{1}, e_{2} \in E .
$$

This implies that $S$ contains a unique idempotent and, since the right cancellation is present, we get by Ljapin [1, p. 113] that $S$ is a group.

REMARK 4. In particular, any finite right cancellation left amenable semigroup is a group (which is well known).

Lemma 5. Let $S$ be a left amenable semigroup for which $S^{\prime}$ is finite and contains $0<n<\infty$ elements (necessarily $S^{\prime}$ is a finite group). Then there is a left invariant mean

(1) Here as in Lemma 5 the condition that $S$ is left amenable can be relaxed to: any two right ideals of $S$ have nonvoid intersection. 
$\phi$ on $m(S)$ for which $\phi(A)$ takes the only values $\{k / n ; k=0,1, \ldots, n\}$, when $A$ ranges over all subsets of $S$.

Proof. Let $S^{\prime}=\left\{s_{1}^{\prime}, \ldots, s_{n}^{\prime}\right\}$ where $s_{i} \in S$ is a fixed representative of $s_{i}^{\prime}, 1 \leqq i \leqq n$ (till the end of this proof). The semigroup $S^{\prime}$ is left amenable as a homomorphic image of $S$ (Day [2, p. 515(c)]) and has right cancellation (if $a^{\prime} c^{\prime}=b^{\prime} c^{\prime}$, then $(a c)^{\prime}=(b c)^{\prime}$, i.e., $a(c s)=b(c s)$ for some $s \in S$; hence, $\left.a^{\prime}=b^{\prime}\right)$. By Remark $4, S^{\prime}$ is a finite group. Assume that $s_{1}^{\prime}$ is the identity of $S^{\prime}$ (otherwise, renumber the $s_{i}$ 's) and let $S_{i}=\left\{s \in S ; s^{\prime}=s_{i}^{\prime}\right\}$ for $i=1,2, \ldots, n$. $S_{1}$ is a subsemigroup of $S$ since if $a, b \in S_{1}$, then $(a b)^{\prime}=s_{1}^{\prime} s_{1}^{\prime}=s_{1}^{\prime}$ and so $a b \in S_{1}$. Furthermore, if $a, b \in S_{i}$ for some $i$, then $a c=b c$ for some $c \in S$. If $c \in S_{j}$, let $s_{k}^{\prime}=\left(s_{j}^{\prime}\right)^{-1}$. Then $\left(c s_{k}\right)^{\prime}=s_{j}^{\prime} s_{k}^{\prime}=s_{1}^{\prime}$; hence, $a\left(c s_{k}\right)=b\left(c s_{k}\right)$ and $c s_{k} \in S_{1}$. We have shown that if $a, b \in S_{i}$, there is some $d$ in $S_{1}$ such that $a d=b d$. In particular, this is true for $a, b \in S_{1}$. Hence, by Corollary 3 of T. Mitchell [5] (for a different proof, see Theorem 3 in [4]), $m\left(S_{1}\right)$ admits a left invariant mean $\phi$ which is multiplicative on $m\left(S_{1}\right)$ (i.e., $\psi(f g)=\psi(f) \psi(g)$ for $f, g \in m\left(S_{1}\right)$ ). Let $\beta(S) \subset m(S)^{*}$ be the set of multiplicative means on $m(S)$ (i.e., which satisfy $\phi(f g)=\phi(f) \phi(g)$ for $f, g \in m(S))$. Then $L_{s}(\beta(S)) \subset \beta(S)$ for any $s$ in $S$ and, in particular, for any $s$ in $S_{1}$. Since $\beta(S)$ is compact hausdorff there exists by Mitchell's fixed point theorem (see T. Mitchell [5] or [4, Theorem 3]) some $\phi_{0} \in \beta(S)$ which is $S_{1}$-left invariant. If $A \subset S$, then $1_{A}^{2}=1_{A}$ and, therefore, $\phi_{0}(A)$ is either 0 or 1 for any $A \subset S$. If $a \in S_{i}$, then $a c=s_{i} c$ for some $c \in S_{1}$ and, therefore:

$$
L_{a} \phi_{0}=L_{a} L_{c} \phi_{0}=L_{a c} \phi_{0}=L_{s_{i} c} \phi_{0}=L_{s_{i}} \phi_{0},
$$

since $\phi_{0}$ is $S_{1}$-left invariant. Furthermore, for any $a \in S$,

$$
\left\{\left(a s_{i}\right)^{\prime} ; i=1,2, \ldots, n\right\}=\left\{a^{\prime} s_{i}^{\prime}, i=1,2, \ldots, n\right\}=\left\{s_{i}^{\prime}, i=1,2, \ldots, n\right\}=S^{\prime},
$$

since $S^{\prime}$ is a group. Let

Then

$$
\phi=\frac{1}{n} \sum_{1}^{n} L_{s_{i}} \phi_{0}
$$

$$
L_{a} \phi=\frac{1}{n} \sum_{1}^{n} L_{a s_{\imath}} \phi_{0}=\frac{1}{n} \sum_{1}^{n} L_{s_{t}} \phi_{0}
$$

which shows that $\phi$ is a left invariant mean. If $A \subset S$, then $\left(L_{s_{i}} \phi_{0}\right)\left(1_{A}\right)$ is either 0 or 1 since $L_{s_{i}} \phi_{0}$ is also multiplicative; therefore, $\phi(A)=(1 / n) \sum_{1}^{n} L_{s_{i}} \phi_{0}\left(1_{A}\right)$ takes one of the values $\{k / n ; k=0,1, \ldots, n\}$. Fix now $1 \leqq i, j \leqq n$ and let $s_{k}^{\prime}=s_{i}^{\prime}\left(s_{j}^{\prime}\right)^{-1}$. If $a \in S_{j}$, then $\left(s_{k} a\right)^{\prime}=s_{k}^{\prime} s_{j}^{\prime}=s_{i}^{\prime}$ which shows that $s_{k} S_{j} \subset S_{i}$. Therefore, for any left invariant $\phi_{1}$ on $m(S), \phi_{1}\left(S_{j}\right) \leqq \phi_{1}\left(s_{k} S_{j}\right) \leqq \phi_{1}\left(S_{i}\right)$. By symmetry, one has $\phi_{1}\left(S_{i}\right)=\phi_{1}\left(S_{j}\right)$. Since $S_{i}$ are disjoint, one has $1=\phi_{1}(S)=n \phi_{1}\left(S_{i}\right)$ or $\phi_{1}\left(S_{i}\right)=1 / n$ for $i=1,2, \ldots, n$. Therefore, if $A_{k}=\bigcup_{1}^{k} S_{i}$ and $A_{0}=\varnothing$ then $\phi_{1}\left(A_{k}\right)=k / n$ for any left invariant mean $\phi_{1}$ on ${ }^{*}$ $m(S)$ and, in particular, the range of the above defined left invariant mean $\phi$ is exactly the set $\{k / n ; k=0,1, \ldots, n\}$. This range is attained on the class of left almost convergent subsets of $S$.

REMARK 5. For each $0<n<\infty$ there is a countable commutative (left) amenable 
semigroup $S$ such that $S^{\prime}$ has order $n$ and each (left) invariant mean on $m(S)$ annihilates single point sets.

Let $T=\{1,2, \ldots\}$ with the multiplication $i j=\max \{i, j\}$ and let $G$ be the cyclic group of order $n$. Then $S=T \times G$ is (left) amenable since it is commutative. Assume that $\left(t_{0}, g_{0}\right) \in S$ is such that $0<d=\psi\left\{\left(t_{0}, g_{0}\right)\right\}$ for some left invariant mean $\psi$ on $m(S)$ and let $e$ be the identity of $G$. Since $\left(t, g_{0}^{-1}\right)\left(t_{0}, g_{0}\right)=(t, e)$ if $t \geqq t_{0}$, we get that $\psi\left\{\left(t_{0}+k, e\right)\right\} \geqq d$ for $k=1,2, \ldots$ which implies that

$$
1 \geqq \psi\left\{\left(t_{0}+1, e\right), \ldots,\left(t_{0}+k, e\right)\right\} \geqq k d
$$

for any $k$. Hence, $d=0$ and any left invariant mean on $S$ annihilates single point sets. It remains to be shown that the order of $S^{\prime}$ is $n$. We show that $a^{\prime}=b^{\prime}$ if and only if $a, b \in(T, g)$ for some $g \in G$. If $a=\left(t_{1}, g\right), b=\left(t_{2}, g\right)$ and $t=\max \left(t_{1}, t_{2}\right)$, then $\left(t_{1}, g\right)(t, e)=(t, g)=\left(t_{2}, g\right)(t, e)$. Hence, $a^{\prime}=b^{\prime}$. Conversely, if $a^{\prime}=b^{\prime}$ and $a=\left(t_{1}, g_{1}\right)$, $b=\left(t_{2}, g_{2}\right)$ then for some $(t, g) \in S,\left(t_{1}, g_{1}\right)(t, g)=\left(t_{2}, g_{2}\right)(t, g)$. Hence, $g_{1} g=g_{2} g$ or $g_{1}=g_{2}$. Therefore, $a, b \in\left(T, g_{1}\right)$. Thus, $\{(T, g) ; g \in G\}$ are the different equivalence classes which give rise to the different elements of $S^{\prime}$. It can be readily checked that $S^{\prime}$ is isomorphic to $G$ in this case.

LEMMA 6. Let $\rho: S \rightarrow T$ be a homomorphism of the left amenable semigroup $S$ onto the semigroup $T$. Assume that there is a family of subsets of $T,\{B(x) ; 0 \leqq x \leqq 1\}$ which satisfies:

(1) $B(0)=\varnothing, B(1)=T$ and $B(x) \subset B\left(x^{\prime}\right)$ if $0 \leqq x<x^{\prime} \leqq 1$.

(2) $\psi(B(x))=x$ for each $0 \leqq x \leqq 1$ and any left invariant mean $\psi$ on $m(T)$.

Then $\left\{A(x)=\rho^{-1}[B(x)], 0 \leqq x \leqq 1\right\}$ satisfies

(1) $A(0)=\varnothing, A(1)=S$ and $A(x) \subset A\left(x^{\prime}\right)$ if $0 \leqq x<x^{\prime} \leqq 1$.

$(2)^{\prime} \phi[A(x)]=x$ for any left invariant mean $\phi$ on $m(S)$ and each $0 \leqq x \leqq 1$.

Proof. It is clear that $A(x)$ satisfy (1)'.

Define $F: m(T) \rightarrow m(S)$ by $(F f)(s)=f(\rho(s))$. Let $\phi \in m(S)^{*}$ be a left invariant mean. Then $F^{*} \phi \in m(T)^{*}$ defined by $\left(F^{*} \phi\right) f=\phi(F f)$ is a left invariant mean on $m(T)$ (see Day [2, p. 515(c) and pp. 531-532]). But $\left(F 1_{B(x)}\right)(s)=1_{B(x)}(\rho(s))=1_{A(x)}(s)$ for each $s$ in $S$. Therefore,

$$
x=\left(F^{*} \phi\right)\left[1_{B(x)}\right]=\phi\left[1_{A(x)}\right]=\phi[A(x)]
$$

for each $0 \leqq x \leqq 1$.

We prove now the main theorem of this paper.

TheOREM 3. Let $S$ be a left amenable semigroup for which $S^{\prime}$ is not an AB group. ( $S^{\prime}$ is necessarily a left amenable right cancellation semigroup which is a group in case it is periodic.)

(1) If $S^{\prime}$ is infinite, then the range of each of its invariant means is the whole $[0,1]$ interval. Moreover, there are, in this case, sets $A(x) \subset S$ such that $A(0)=\varnothing$, $A(1)=S, A(x) \subset A\left(x^{\prime}\right)$ if $0 \leqq x<x^{\prime} \leqq 1$, and $\phi(A(x))=x$ for each left invariant mean $\phi$ on $\dot{m}(S)$. (In particular, $\phi$ attains its range on left almost convergent sets.) 
(2) If the range of each left invariant mean on $m(S)$ is the whole $[0,1]$ interval, then $S^{\prime}$ is infinite.

Proof. We first prove (2). If $S^{\prime}$ contains $0<n<\infty$ elements, then by Lemma 5 there is a left invariant mean $\phi$ on $m(S)$ whose range is the set $\{k / n ; k=0,1, \ldots, n\}$. (There are even $A_{i} \subset S$ with $\varnothing=A_{0}, A_{n}=S, A_{i} \subset A_{i+1}$ for $0 \leqq i \leqq n-1$ and such that $\phi\left(A_{k}\right)=k / n$ for $k=0,1, \ldots, n$ and each left invariant mean $\phi$ on $m(S)$.)

We prove now (1):

(a) If $S^{\prime}$ contains an element of infinite order, then apply the corollary to Theorem 1 and Lemma 6.

(b) If $S^{\prime}$ does not contain elements of infinite order, then $S^{\prime}$ is periodic which implies by Lemma 5 that $S^{\prime}$ is a group which by our assumption contains an infinite locally finite subgroup $T_{0}^{\prime} \subset S^{\prime}$. There are now finite subgroups $S_{n}^{\prime} \subset T_{0}^{\prime}$ with $S_{n \nsubseteq}^{\prime} S_{n+1}^{\prime}, n=1,2, \ldots$ Let $S_{0}^{\prime}=\bigcup_{1}^{\infty} S_{n}^{\prime}$. As a locally finite group, $T_{0}^{\prime}$ is

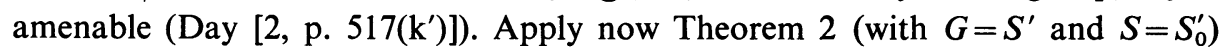
and Lemma 6.

Consequence.

(a) If $S$ is a semigroup which admits a left invariant mean $\phi$ for which $\phi(A)$ $\neq(1 / \sqrt{2}$ ) (say) for each $A \subset S$ (or even for each left almost convergent $A \subset S$ ), then $S^{\prime}$ is either an $A B$ group or a finite group.

(b) If $S^{\prime}$ is not an $A B$ group, then the set $\{\phi(A) ; A$ ranges over all left almost convergent subsets of $S\}$ is either the entire [0,1] interval for each left invariant mean $\phi \in m(S)^{*}$, or the set $\{k / n ; k=0,1, \ldots, n\}$, for some $0<n<\infty$, for each left invariant mean $\phi \in m(S)^{*}$.

(c) Is there a finitely additive translation invariant measure $\phi$ on the set of all subsets of the additive integers $Z$ such that $\phi(Z)=1$ and $\phi(A)$ is rational, say, for any $A \subset Z$ ? Any such $\phi$ is, as is easily checked, the restriction of some invariant mean $\psi$ on $m(Z)$, to $\left\{1_{A} ; A \subset Z\right\}$. Theorem 3 implies hence that the answer to this (and in fact to a much more general) question is negative.

\section{REFERENCES}

1. E. S. Ljapin, Semigroups, Transl. Math. Monographs, Vol. 3, Amer. Math. Soc., Providence, R. I., 1963.

2. M. M. Day, Amenable semigroups, Illinois J. Math. 1 (1957), 509-544.

3. E. Granirer, A theorem on amenable semigroups, Trans. Amer. Math. Soc. 111 (1964), 367-379.

4. —- Extremely amenable semigroups, Math. Scand. 17 (1965), 177-197.

5. T. Mitchell, Fixed points and multiplicative left invariant means, Trans. Amer. Math. Soc. 122 (1966), 195-202.

6. G. G. Lorentz, A contribution to the theory of divergent sequences, Acta Math. 80 (1948), 167-190.

University of British Columbia, Vancouver, British Columbia, Canada 